\title{
Le Goût de la guerre
}

\author{
Catherine Mavrikakis, Université Concordia \\ Martine Delvaux, UQAM
}

\begin{abstract}
Through an exchange of letters, the authors of this piece discuss the event of September 11 and its representation - in particular, questions of rumour and silence, witnessing and responsibility. The issue of gender and the "feminization" of the event are specifically considered through a reading of the appeal not only to our senses but also to our capacity for insensitivity.
\end{abstract}

Montréal, le 4 octobre 2001

Ma chère Martine,

Ça parle sans cesse, ça n'arrête pas de parler, ça cause, mais qu'est-ce que ça cause. Ça piaille, ça crie, ça dit n'importe quoi. Il n'y a que de la rumeur. De la fausse rumeur. Ça n'arrête pas. C'est abasourdissant. J'en ai la tête toute gélatineuse. J'en ai les oreilles farcies. Sur CNN, ABC, NBC, CBS, toute la journée, à toutes les heures. Ça parle tout le temps. Et des noms qui résonnent, des noms qui claquent comme des portes qu'on ferme. Peter Jennings, Aron Brown, Paula Zahn, Larry King, WTC, Ground Zero, Afghanistan, Islamabad, Mohammed Atta, Hillary Clinton, Giuliani, Bush, Powell, Rumsfeld, Pentagone, Flight 93 et un nom, un nom qui nous tambourine les tympans, un nom qu'on va entendre jusqu'à la nausée. Oussama ben laden. Oussama ben laden. Je zappe. Oussama ben laden. J'ai baptisé ma fille ces derniers jours, Oussama ben Loulou. Je ne voulais pas qu'elle soit en reste. Je voulais qu'elle aussi participe à la rumeur mondiale. Au bruit de fond de la bêtise universelle.

Il fait silence en ce moment. Entre nous, et dans le monde. J'essaie pourtant de faire parler la rumeur du monde. De lui permettre du sens, à cette rumeur folle, cette rumeur affolée. Les cris des morts dans la nuit, les morts passés et à venir, mais tout cela ne me dit rien. Les morts n'ont pas besoin de moi, pas en ce moment. Et les vivants me sont comme une blessure. 
Montréal, le 6 octobre 2001

Chère Catherine,

Le 11 septembre est venu interrompre notre correspondance, $l^{\prime}$ attaque terroriste contre les Etats-Unis est venu parasiter notre conversation, celle-là et tout le brouhaha quotidien qui fait du bruit dans nos vies, comme si on s'était perdues d'ouïe...

J'ai donné un coưrs, jeudi après-midi, sur les Lettres parisiennes de Nancy Huston et Leilla Sebbar, ce texte qui a motivé le début de notre échange. J'en ai été, comme la première fois, émue, animée, car ces lettres sont belles, vivantes. Mais j'ai été aussi bouleversée durant la préparation de ce cours, comme si on m'avait giflée. Fouillant les textes de Huston et Sebbar, lisant à la fois l'une et l'autre, sur l'une et l'autre, je me suis rendue compte qu'elles avaient toutes deux mis en scène un même événement dont, honteusement, je ne connaissais pas l'existence: l'enfermement, la torture, le meurtre d'Algériens à Paris le 17 octobre 1961. Des centaines sont morts alors qu'au lendemain du carnage, on annonçait le chiffre officiel de deux disparus. La Seine était rouge, écrit Sebbar, la Seine dans laquelle ont été projetés les corps mutilés de protestants pacifiques au couvre-feu déclaré par Papon. A l'époque, on a interdit aux média de faire état de l'événement, on l'a mis sous silence, on a tenté de l'effacer. Ce n'est que vingt ans plus tard que la mémoire a été réanimée. Huston en parle dans $L^{\prime} E m p r e i n t e$ de l'ange. J'en ai parlé à mes étudiants qui écoutaient, sidérés. Comment aije pu ne rien apprendre de cette histoire durant toutes ces années? Quel écran, quel filtre s'est installé? La rumeur, dans ce cas-là, n'a pas fonctionné. Rien n'est venu à mes oreilles. Je n'ai rien entendu.

Comme toi, je suis étourdie par la suite infinie de noms, d'aphorismes, d'avertissements, de menaces, de prières que les média font pleuvoir sur nous. Et pourtant, ça demeure, pour moi, le lieu du silence. Il y a quelque chose de terrible dans la façon dont la représentation télévisée, en cherchant à rendre les événements sur le vif, vide la réalité de ses sens. Quand je vois l'avion percuter la seconde tour du World Trade Center, je n'entends rien. Quand je vois les secouristes, les travailleurs s'affairer à Ground Zero, mes oreilles sont bouchées, comme si les couches de débris, l'épaisseur de la poussière, tissaient un immense linceul non seulement autour des victimes mais autour des témoins. Je vois mais je n'entends pas. La rumeur fait silence. Elle nous force à ne pas entendre. Elle nous force à oublier.

Oussama ben laden, les réfugiés afghans, les Marines qui se marient avant le grand départ, les manifestations au Pakistan, l'émotion de Rudolf 


\section{Le Gout de la guerre $\cdot 65$}

Giuliani...Oui, il y a foule dans nos oreilles. Et quand tout le monde parle personne ne parle, comme dirait Marguerite Duras, alors que quand quelqu'un parlé, c'est qu'il se prend pour le maître, qu'il prend la parole et interrompt la rumeur. Je crois préférer cette rumeur à la parole d'un George Bush, car il y a de la place dans la rumeur, il y a malgré tout de la place pour respirer, pour entendre autre chose, même si c'est ce qu'on veut bien entendre, ce qu'on espère voir surgir dans les interstices du bruit. On peut entendre plein de choses dans le bruit, alors que la parole unique porte admirablement bien le silence.

Mais quand je pense au 11 septembre, je pense surtout à l'odeur, je l'imagine, je la sens, comme je sens l'odeur du sang à la surface de la Seine.

\section{Montréal, le 7 octobre 2001}

Chère Martine,

Les événements se précipitent sans que nous sachions où tout cela nous mènera. Aujourd'hui, l'Afghanistan est bombardée. La terre tremble de peur. Je ne connais toujours pas le bruit des missiles. On n'arrête pas de nous les montrer à la télé, mais on ne m'a pas dit le bruit des corps qui se déchirent, le bruit de la peur au ventre, le bruit de la mort. Il paraît que lorsque l'on a très peur, on défèque sans s'en rendre compte. Nous n'avons pas encore connu une défécation collective. $\mathrm{D}^{\prime}$ autres pays connaissent cela. L'odeur de la merde de tout un peuple. L'odeur du cadavre de tout un peuple. Nous, Martine, filles de l'après-guerre au Canada, nous ne connaissons pas l'odeur de chair brâlée ou encore l'odeur de la putréfaction de nos corps. Et nous préférerions ne pas apprendre. Nous préférerions l'ignorance. Est-ce là notre culpabilité ou encore notre innocence face à la vie? Il y a la mémoire. Il y aura la mémoire de ce qui sera sans doute oublié. Mais ce n'est pas dans quarante ans que l'on s'étonnera de ne pas savoir. C'est maintenant que je m'étonne de mon ignorance feinte. Ce n'est, bien sûr, pas la Seine qui est rouge aujourd'hui, mais je ne pourrais pas dire qu'elle ne l'est pas. Mes mains sont peut-être tachées de mon sang ou de celui des autres. Et le monde entier voit rouge. C'est maintenant que je suis coupable ou innocente de ne pas savoir ou de ne pas vouloir savoir. $C^{\prime}$ est maintenant que je préfère fermer les yeux sur tout et ne pas connaître dans ma chair le goût de la guerre.

Ce soir, le Canada est du côté des "alliés". Même la Chine a donné son accord à des tirs ciblés sur l'Afghanistan. Et je me demande, sûrement bêtement où le Québec est. Où le Québec serait, s'il pouvait parler. De cela aussi, comme du reste, ce soir, j'ai si peur, Martine. 
Montréal, le 8 octobre 2001

Chère Catherine,

Je n'ai jamais compris d'où pouvait venir ce goût de se battre pour son pays. Et hier soir, en regardant la télévision, je n'ai pas compris ce qui motivait ces gens qui, à New York, venaient opposer leur manifestation à celle qui avait lieu pour la paix. Ils qualifiaient de traitres leurs compatriotes qui prenaient position contre la guerre. D'où vient cette folie, ce goût pour la chair ensanglantée? Ou n'est-ce pas que la réalité de la guerre se trouve à des lieux de distance de nos communautés privilégiées? De là le succès, peut-être, du Saving Private Ryan de Spielberg qui amenait au plus proche de nous les corps déchiquetés sur les plages de Dieppe, si proche qu'on s'en croyait éclaboussés, si proche qu'on pouvait croire partager avec eux la surdité de la mer, l'épaisseur de l'eau noire de sang.

Moi aussi, j'ai peur, d'une peur qui doute d'elle-même, d'une peur un peu informe qui demeure malgré tout abstraite. Car de quoi ai-je peur, au juste? Ai-je peur de la douleur qui sera celle des Afghans au pire de ce conflit? Ai-je peur de leurs pieds endoloris, de leurs muscles épuisés dans cette marche hors de leur pays? Ai-je peur de leur faim, de leur soif de réfugiés, du soleil brûlant sur leur peau, ou du froid des nuits? Est-ce que je crains, comme s'il s'agissait de ma propre vie, l'incertitude, la violence, la maladie qui sont le lot de ceux qui fuient? Quelles sont les limites de mon empathie? Ma peur est-elle autre chose qu'égoiste? Ne devient-elle pour moi réelle que quand je m'imagine dans un aéroport aux Etats-Unis? Qu'est-ce que je veux dire, moi, québécoise, face à cette guerre? Et qu'estce que le Québec dira en attendant qu'il se trouve vraiment interpellé?

"Nous n'avons pas commencé cette guerre" disait hier cette Américaine pro-guerre. C'est maintenant qu'ils se savent réellement attaqués, alors qu'ils attaquent sans cesse depuis une éternité, que les Américains se mettent à parler, à dire cet événement. Mais comme le suggère Derrida, si on peut dire un événement, $c^{\prime}$ est qu'événement il n'y a plus vraiment, car un événement, s'il est vraiment événement - surprise, étonnement, apparition, révélation - , ne se donne pas à dire. Dès qu'il se dit, l'événement n'est plus événement, ou bien il est un nouvel événement, un événement qui se fait, car le dire l'événement est toujours un faire l'événement plutôt qu'un rendre compte de l'événement. Je me demande donc quel événement nous sommes en train de faire, de fabriquer? Quel événement nous inventons pour être prêts à y croire? 
Je ne me battrais jamais pour mon pays, je ne revêtirais pas d'uniforme, je ne prendrais pas les armes, je ne pointerais pas de fusil contre la tempe d'un ennemi, car la nation est un événement auquel je ne crois pas, au profit duquel je ne ferais pas d'acte de foi. Une nation se construit toujours sur des tranchées, un charnier, une fosse commune. Le nouveau monde $s^{\prime}$ évertue à oublier les squelettes sur lesquels il s'est bâti. Si on regardait bien, on verrait peut-être ce que l'effondrement des tours du World Trade Center nous met sous les yeux. Six mille corps réduits en miettes. Six mille corps qui représentent tous ceux sur lesquels reposait un empire.

George Bush descend de son avion. Un militaire l'acceuille au pied de l'escalier, le salue de sa main gantée de blanc. Les taches de sang sont invisibles.

\section{Montréal, le 12 octobre 2001}

Les jours passent, Martine, les jours passent gros d'eux-mêmes, uniquement d'eux-mêmes. Les dates me terrifient. J'ai toujours peur le matin en me réveillant que le bruit de la journée qui s'amorce soit aussi le son abrutissant que ferait le glas.

Pour qui sonne le glas? Pour quoi sonnera-t-il, demain? L'Afghanistan, c'est vraiment bien loin. Les missiles, les bombardements dans la nuit, la faim, la peur, que c'est abstrait tout cela pour un Montréalais du centreville ou de la banlieue lavalloise...Et même New York, tu vois, des milliers de morts évanouis en fumée, c'est presque un concept: l'injustice ou quelque chose du genre. Ce n'est rien de bien clair. Rien que l'on puisse vivre vraiment. Sur la caméra fixe de Kaboul, tu peux voir à la télé des explosions de temps à autre. C'est une couleur verte dans la nuit. On voit mal, ce n'est pas net. Ce ne sont que des gens qui meurent dans le vert psychédélique des bombes à l'écran.

Alors quoi? Qu'est-ce qu'il faut que Montréal vive dans sa chair? Pourquoi cette panique devant une enveloppe au centre-ville, pourquoi un périmètre de sécurité et cent voitures de police au coin de Crescent et Sherbrooke, à la sortie de mon cours mardi? Pourquoi cela devrait-il nous toucher, comme cela? Quelle est cette idée que cela devrait se marquer sur nos corps? Nos esprits, notre intelligence, notre pensée ne sont-ils pas suffisamment atteints? De quel refoulement s'agit-il, pour que nous voyions la peur à tous les coins de rue; alors que d'autres ailleurs meurent réellement et sans pouvoir alerter qui que ce soit? Faut-il que cela nous frappe en plein coeur pour que nous sentions la douleur? Sommes-nous à ce point 
frigides? Que demandons-nous encore pour que nous nous mettions à voir quelque chose ou à avoir dans la bouche le goût exacerbé des chairs malades ou calcinées? Faut-il que nos chairs soient en lambeaux épars sur le sol de la ville, pour que nous ayons l'impression que quelque chose s'est vraiment passé et que ce n'est pas seulement à venir?

Je te le dis: quelque chose a eu lieu. Mais cela ne nous suffit pas. Il faut que cela se répète chez nous, en nous. Et serait-ce suffisant?

La peur...cela nous permet d'oublier ce qui vient de prendre place sous nos yeux, d'oublier les frappes en Afghanistan et les gens qui hurlent loin, bien loin...De peur.

Quelque chose nous échappe, bien sûr, mais pourquoi vouloir retenir à tout prix ce qui fuit sur le mode du "nous aussi, nous allons y goûter"? N'y a-t-il pas d'autre moyen de se saisir de ce qui a eu lieu?

Je pense, comme je te l'ai écrit ailleurs, à la couleur de la peau, à la couleur des yeux, à nos noms, nos accents, nos langues maternelles, nos langues étrangères performées ou balbultiées. Je pense au fait très simple que je ne suis pas arabe, que peu de gens dans le monde pourraient croire que je le suis. C'est comme cela, j' ai l'air d'une occidentale. Par mon air, ma voix, ma façon de parler. Je ne suis pas non plus musulmane et cela se voit aussi. Cela se voit, ces choses-là.

Aux États-Unis, un homme a tué un Sikh parce qu'il portait un ruban et qu'il ressemblait pour un Américain très moyen à un terroriste musulman. Là encore, ce serait l'ironie du sort. Les Sikhs détestent les Musulmans, alors vraiment, c'est presque drôle cette mort ridicule survenue à cause $\mathrm{d}^{\prime}$ une mauvaise connaissance du géopolitique.

Mais il n'y a pas d'erreur sur la personne. Les tueurs ne se trompent jamais...On tue ce qui ne nous ressemble pas. On ne fait pas dans les détails. Ce n'était qu'un sale étranger à turban. Ce n'était rien, ce n'était pas "nous".

On ne choisit pas à quoi on ressemble. On ne choisit pas d'être pauvres ou malheureux. On ne choisit pas d'être une femme en Afghanistan sous le régime des Talibans. On ne choisit pas de porter le voile là-bas, ou encore de se plonger sous le tchadrié. Il y a des choses qu'on ne choisit pas.

Il y a des choses qu'on ne peut pas choisir.

Dans certains pays, on ne choisit pas de mourir pour sa patrie. On n'a pas le choix. On meurt. L'autre jour, une femme afghane racontait comment depuis quelques années, dans la clandestinité, elle éduque et instruit les femmes de son pays. Elle risque la mort chaque iour. varce au'elle 
croit à un avenir pour les femmes de son pays. On ne choisit pas cela. Elle n'avait pas choisi.

On ne choisit pas.

Néanmoins, un journaliste français a cru qu'il pouvait choisir. Il s'est déguisé en femme pour aller en Afghanistan mener l'enquète. Sous le voile, tout le monde est pareil, non?

Si un homme venu d'ailleurs s'était mis en mini-jupe et avait épousé tous les codes de la féminité pour ne pas être reconnu dans un de "nos" pays, qu'est-ce que nous aurions rigolé de lui, ici, nous les citoyens du monde. Quelle naiveté, aurions-nous dit, face à nos coutumes, au marquage de la différence sexuelle! Quelle ignorance de notre conception de la féminité! Et même les plus constructivistes d'entre nous, ceux et celles qui croient que la différence sexuelle est culturelle, nous nous serions bien amusé de cet homme.

Mais vois-tu, il nous faut un tel aveuglement face au monde musulman pour avoir le culot de croire qu'il suffit de porter un voile pour être une femme aux yeux des Talibans. Il nous faut une telle bêtise pour ne pas voir ce qu'eux voient. Cela doit nous prendre pas mal d'énergie pour arriver à ne rien voir, à ne rien savoir des autres. La seule empathie que nous ayons, c'est celle de la peur que nous voudrions sentir dans nos chairs, et toujours un peu plus. "Fais nous encore peur avec anthrax, Kaboul est si loin..."

Il faut sûrement penser toute la journée aux enveloppes pleines d'anthrax pour ne pas voir Kaboul en flammes, pour ne pas sentir l'odeur de la mort qui a déjà eu lieu, pour ne pas entendre les cris des Palestiniens, depuis tant d'années, et pour oublier déjà les morts du 11 septembre en nous précipitant nous-mêmes sur le manège de la peur. Cela doit prendre un sacré effort, non.

Occidentaux, encore un effort avant d'être complètement cons....Ce n'est plus très loin, la victoire...

Il est possible que nous mourions un jour à cause du simple fait que nous étions occidentales. D'autres, ailleurs, sont morts, d'être nés au mauvais endroit. Nous ne pourrons pas brandir le fait que nous avions quelques amis musulmans le jour où l'on fera sauter notre avion. Je ne pourrai pas dire très rapidement, glisser que je suis de gauche, pro-palestinienne et que j'ai espéré mieux pour l'Occident. Beaucoup mieux. Je ne pourrai rien dire. Je ne saurai que me taire. Je contemplerai le désastre, à l'occidentale. 
Montréal, le 15 octobre 2001

Chère Catherine,

Depuis ma table de travail, je reçois des informations sur une guerre qui se déroule loin d'ici, sur un territoire dont la géographie physique éveille ma fibre touristique, dont les images me sont sûrement exotiques, jusqu'au vêtement de ses habitants. Le burqa bleu des femmes accueillant des dignitaires étrangers, une image que j'ai vu plusieurs fois à la télé et qui me laisse perplexe. Ce burqa qui est peut-être l'élément central, pour moi, de la propagande entourant cette guerre. Bien avant les événements du 11 septembre, on avait vu circuler des pétitions contre le traitement fait aux femmes par les Talibans. Maintenant que les tours se sont effondrées, ce qui jusque là ne valait pas la peine d'être combattu devient le prétexte à une guerre insensée. C'est sur le dos des femmes que ça se fait. C'est sur leur histoire que prend appui le discours américano-occidental pro-guerre anti-taliban. Je me suis souvent dit que s'il y avait une bonne chose à tirer de cette guerre, c'était la levée du voile sur la situation des femmes en Afghanistan. Mais cet exemple du journaliste français "déguisé" montre bien à quel point cette situation sert simplement de porte d'entrée... .

J'ai voulu pleurer, de rage, quand j'ai entendu Oprah Winfrey dire à une femme afghane au visage caché, qui suffoquait sous ses larmes alors qu'elle racontait s'être enfuie après avoir été emprisonnée par les Talibans pour avoir servi d'interprète à une journaliste américaine (alors que ce sont les Talibans qui lui avait assigné ce travail): "C'est fini, maintenant. Vous êtes arrivée aux Etats-Unis." On aurait dû faire porter à cette jeune femme un voile fait du drapeau américain...Son regard agard, effarouché, indiquait bien qu'on n'y comprenait rien...Je me suis dit qu'il y avait un montant d'argent à la clé et j'ai souhaité que ça lui permette de vivre un peu mieux. Puis j'ai pensé à tous ces réfugiés afghans, à tous ces gens qui cherchent à entrer dans un pays, aux Etats-Unis par exemple, et qu'on accueille difficilement, à qui on ne donnerait pas une miette de pain. $C^{\prime}$ est sur le dos de cette femme qu'on se donne bonne conscience.

J'ai une fois croisé, dans le métro Jean-Talon, en direction de Snowdon, une femme recouverte du burqa. Cette fois-là aussi une vague de colère m'a envahie, je me suis sentie vaciller, je n'ai pu m'empêcher de fusiller du regard l'homme qui l'accompagnait qui, lui, ne m'a pas du tout regardée. Les mots de multiculturalisme, d'immigration, d'intégration ont avec fulgurance traversé mon esprit. J'étais entre la rage et la culpabilité. Mais je crois qu'il s'agit ici d'autre chose que des petites filles de Creil. Le foulard, 
ce n'est pas le burqa. Je ne supporte pas cette cage vestimentaire qui paralyse et force l'anonymat. Je ne supporte pas de voir ces femmes au regard effacé, amputé, qui ne peuvent pas voir autour d'elles. Ce n'est que devant qu'elles peuvent regarder.

Et nous, que voyons-nous devant nous? D'où vient notre incapacité à être témoin? Il faudrait mimer chez nous l'événement pour croire vraiment qu'il a eu lieú. Un drôle d'accompagnement qui sert bien toute une mécanique du déni, de l'aveuglement. On se met sous le voile puisque de toute façon on échoue à la vision. Ce n'est pour rien excuser, mais je me dis parfois que l'effet de la surenchère iconographique est d'annuler la capacité à être empathique. Comme si on perdait prise sur la réalité. C'est une façon très bête de se rapprocher d'un événement, de se joindre à la "fête". - ça dit "moi aussi je souffre, moi aussi j'ai peur, moi aussi je suis de la partie"...

Un écureuil albinos vient d'apparaître dans ma fenêtre. Il s'approche et me dit que nous sommes blancs, occidentaux, et bien incapable de regarder.

Montréal, le 16 octobre 2001

Chère Martine,

Parfois, dans cette histoire, j'ai souvent l'impression qu'il ne faut plus compter sur nos sens pour penser cet événement. Nos yeux sont aveuglés, nos oreilles bouchées, nos bouches hantées par les bactéries annoncées, nos nez altérés par l'anthrax. Nos paroles sont des clichés, des redites, des imitations grotesques qui ne parviennent pas à dire le désastre. Nos sens sont manipulés et nous participons à cette entourloupette générale. Mais que faire d'autre? Tu as raison. Que faire d'autre, que des pitreries? Aucun témoignage ne peut venir dire quelque chose, parce qu'ici nous ne pouvons que nous laisser aller à nos sens, à l'empathie, à l'expérience qui a toujours raison, contre tout, mais qui mène aussi au pire. Que dire devant l'expérience physique ou mentale de la catastrophe? Hier, j'étais avec les pompiers New-Yorkais qui pleurent en évoquant le bruit terrible que font les immeubles quand ils s'effondrent. Je ne savais pas le grondement de l'horreur, je n'avais jamais entendu parler du silence après l'écrasante cavalcade de millions de tonnes sur le sol. J'ai partagé pendant un moment le murmure terrible de la mort au World Trade Center.

Demain, je serai avec la mère d'un jeune Palestinien qui se sera fait descendre au coin d'une rue. J'apprendrai le froid du corps de l'enfant 
reur, dans la réalité des corps brûlés, écrasés, morcelés...mais dans l'énergie qui se déploie contre le pouvoir institutionnalisé. Peut-être que j'ai déjà souhaité "tirer au hasard dans la foule", comme le proposait André Breton. Demain, je parlerai à des Américains de la question de la transgression, je leur proposerai de ne pas transgresser, de refuser cette loi qui permet de maintenir la loi, cette loi qui est celle de la transgression. Je leur proposerai de ne pas choisir entre l'espoir vain d'une transgression et la terreur vaine d'une loi. Mais au fond, est-ce que j'y crois?

A l'aéroport de Cleveland, je cherche les traces du désastre, les marques de cette transgression des transgressions qu'a été l'attaque du 11 septembre dernier. Je cherche mais je ne trouve que des gens ordinaires, des étudiantes au tein de pêche, des femmes d'affaires mal habillées, des hommes accrochés à leur téléphone cellulaire...J'ai l'impression d'avoir rêvé. Je me trouve tout à coup très bête de m'être attendue au pire, d'avoir attendu le pire comme une preuve incontestable de la réalité. Les mots de George Bush ont été entendus: les Américains continuent à vivre, à dépenser, à manger de la pizza, à écouter des CD. Et moi, j'en suis à me demander où se trouve la réalité, si ce que je vois, ce que j'appréhende avec les sens, c'est bien ça. La vie est un songe, et même mon corps, qui pourtant devrait être le témoin ultime, n'a plus la capacité de me convaincre. Mes sens m'inspirent la méfiance.

De Montréal, je pouvais imaginer une Amérique catastrophique et catastrophée, et maintenant que j'y suis, je ne vois pas ce que je croyais y trouver. Demain, je vais parler du 11 septembre, faire la liste de mes craintes, énumérer mes fantasmes, me faire honte, peut-être, en parlant de ce qui m’apparaît maintenant comme une Amérique mythique. Mais peut-être aussi que l'Amérique que je crois voir en ce moment, calme, normale comme cet aérogard lumineux depuis lequel je t'écris, est elle aussi un rêve? Peut-être qu'on ne peut véritablement rien voir, qu'on ne peut jamais voir véritablement? Peut-être qu'on ne peut que perdre l'ouïe, la vue, l'odorat, le toucher, même le goût lorsqu'on se trouve devant la catastrophe. Peut-être que c'est ce à quoi Derrida veut en venir lorsqu'il ditqu'on ne peut pas dire l'événement. Pour être un événement, l'événement devrait sans cesse avoir lieu et toujours une première fois, sans jamais pouvoir se trouver dit, écrit, capté, représenté, sans jamais être vraiment senti. C'est peut-être pour ça que les fausses alertes se multiplient, que chacun crie au secours, comme si on voulait de toutes les façons possibles faire vivre l'événement, l'incarner, le ressusciter par le biais de notre pro- 
pre chair. Mais ce faisant, on ne le sent pas, on n'y goûte pas, pas vraiment, puisqu'il s'agit toujours d'un autre événement, d'un événement différent.

Le goût de la guerre, le goût du sang... Le 17 octobre dernier, le maire de Paris, Bertrand Delanöé, a inauguré une plaque pour commémorer que la Seine a été en sang. Il y a eu des opposants à ce devoir de mémoire, des gens qui ont manifesté de leurs corps contre la résurgence du souvenir. Entre 30 et 200 mórts qu'on aurait préféré oublier...Nous sommes durs d'oreille, et nous préférons souvent demeurer muets plutôt que de parler. Mais comment nos corps peuvent-ils si facilement se sentir immunisés, être tétanisés devant la douleur des autres qui sont comme nous? Notre survie est-elle si fragile, et notre santé, notre sanité?

Je te laisse, Catherine, au son des nouvelles de Radio-Canada que je retrouve à mon retoúr, et où le journaliste décrit la difficulté de mettre le doigt sur les Talibans, de les "enfumer" comme dit le plus grand des grands, le chef d'un "nous" bien petit et bien insensé.

En attendant la suite des événement, je t'envoie une grosse bise ainsi qu'à Oussama ben loulou. 